\title{
Observing High-Energy Sources with REM: A Facility for Fast GRB Follow-up
}

\author{
P. Ward and E.J.A. Meurs (on behalf of the REM collaboration) \\ Dunsink Observatory, Castleknock, Dublin 15, Ireland
}

\begin{abstract}
REM is a fast slewing automatic telescope dedicated to the prompt observation of GRB afterglows. The telescope automatically reacts to GCN alerts, beginning follow-up observations in both infra-red and optical wavelengths. Recent observations of GRBs have shown the capabilities of REM to begin follow up observation in the order of 10 s of seconds after the burst.
\end{abstract}

Keywords. gamma rays: bursts, instrumentation: miscellaneous.

REM is a fully robotic fast slewing telescope hosting a near infra-red imaging camera covering the 0.95-2.3 $\mu \mathrm{m}$ range and optical imager/spectrograph covering the range 0.45 $0.95 \mu \mathrm{m}$. With such instrumentation REM is an extremely useful facility for a broad range of applications. It is REMs objective to observe GRBs simultaneously in both the optical and IR. For about half of the bursts with an X-ray afterglow an optical afterglow has been detected as well. In these cases the monochromatic flux decreases in time as a power law $F_{\nu}(t) \sim t^{\delta}$ with $\delta$ in the range $0.8-2$. The first detection of an afterglow made the community realise that a significant amount of information about GRB physics is contained in the early afterglow.

Below are the initial results of GRB observations made by the REM telescope. In some cases the burst was not visible for a number of hours from the telescope site.

GRB050607 - Observations were performed in fully automated mode simultaneously in the near infrared and in the optical, starting approximately $\mathbf{4 7}$ seconds after the alert. Two R-band 3s exposures and five H-band 10s exposures confirm a non-detection, from which upper limits at 3 sigma level of $\mathrm{R}=17.1$ and $\mathrm{H}=16$ can be derived. Magnitudes have been calibrated with a set of stars around the position of the optical detection (GCN 3527) extracted from the 2MASS catalog for the $\mathrm{H}$ filter and from the USNO B1.0 catalog for the $\mathrm{R}$ filter.

GRB050509c - Observations of GRB 050509 were performed approximately $\mathbf{5 6}$ seconds after the burst (32 seconds after the reception of the burst alert). At 2.5 hours after the burst no object was found at the position of the candidate down to a limiting magnitude of $\mathrm{R}=19$ and $\mathrm{H}=15.5$ (3-sigma level). We note that our $\mathrm{R}$ limit is consistent with the light curve power-law index observed by Gorosabel et al. (GCN 3425).

GRB050408 - The field was imaged with both REM instruments in V, R, I, J, H and Ks filters starting at 00:31 UT (approximately 8.2 hours after the burst) for a total integration time of 200 seconds for each filter. No sources are detected within SWIFT XRT error circle (Wells et al., GCN 3191) and at the position of the Optical Transient (Ugarte et al., GCN 3192; Huang et al., GCN 3196) down to a limiting magnitude of 18.5, 18.3, 17.9, 16.8, 17.4, 18.4 (5-sigma upper limit) for V, R, I, J, H, and K filters.

GRB050306 - The field was imaged in R, J, H and Ks filters starting at 8:28 UT (approximately 28.9 hours after the burst). Total integration time was 32, 84, 84 and 94 seconds respectively. A quicker observation could not be performed before due to the 
Moon proximity. Comparison with USNO B1.0 and 2MASS catalogues did not reveal new sources down to a limiting magnitude of $\mathrm{R}>17.0, \mathrm{~J}>15.4, \mathrm{H}>15.2$ and $\mathrm{Ks}>$ 15.0 (3-sigma upper limit).

GRB050209 - Observations were performed in the V, R and I filters (REM IR camera was in maintenance). Observation started on 2005 Feb 09.163 UT, approximately $2.4 \mathrm{~h}$ after the GRB. The field was imaged in each filter for a total exposure time of 400s, under good seeing conditions. No new sources were detected within the HETE error circle by comparison with the Digitized Sky Survey, down to limiting magnitudes $\mathrm{V}=19.1, \mathrm{R}=19.1$, $\mathrm{I}=18.5$ (5-sigma limits).

GRB050128 - Optical observations were carried out in V, R, I filters starting on 2005 Jan 28, at 7:10 UT, and ending at 9:45 UT (approximately 3 hours after the burst). Observations have been partly affected by the presence of the bright Moon close to the field, so only frames with short exposure time can be used. The field was imaged in each filter for a total exposure time of 50s. No new sources were detected within the XRT error circle (Antonelli et al., GCN 2991) down to limiting magnitudes of $\mathrm{V}>18.2, \mathrm{R}>$ 18.2 and $\mathrm{I}>17.9$.

GRB041224 - Observations were carried out in the J, H and $\mathrm{K}$ filters, starting on 2004 Dec 25, at 1:06 UT, and ending at 1:39 UT; approximately 5 hours after the burst. Visual inspection of the NIR frames didn't reveal any new object when compared with the 2MASS catalog.

GRB041223 - Observations were carried out on 2004 Dec 24 from 02:37 UT to 03:11 UT; about 12 hours after the burst. The comparison with the 2MASS catalog did not reveal new infrared sources with $\mathrm{S} / \mathrm{N}>5$ at the limit of the catalog. In particular no source is present at the position of the X-ray afterglow detected by Swift-XRT (Burrows et al. GCN 2901) and of the optical transient reported by Berger et al. (GCN 2902) and Malesani et al. (GCN 2903). The optical observations did not show any convincing candidate either. The 3-sigma upper limit in the $\mathrm{R}$ band is $\mathrm{R} \sim 18$.

Initial results with REM, since commissioning phase, have shown the telescope's ability to begin automated observations within 10 s of seconds of the burst trigger. In the case of the most recent bursts, GRB050607 and GRB050509c, observations were performed simultaneously in the near infrared and optical starting approximately $\mathbf{4 7}$ and $\mathbf{3 2}$ seconds, respectively, after the alert.

\section{References}

Antonelli, et al., GCN 2991

Berger, et al., GCN 2902

Burrows, et al., GCN 2901

Gorosabel, et al., GCN 3425

http://www.merate.mi.astro.it/docM/reports/ann2002/annuario/rem/node3.html http://gcn.gsfc.nasa.gov/gcn3_archive.html

Malesani, et al., GCN 2903

Rhoads, et al., GCN 3527

Wells, et al., GCN 3191 